\title{
Research on the Capacity of Li-ion Battery Packer Based on Capacity Incremental Curve
}

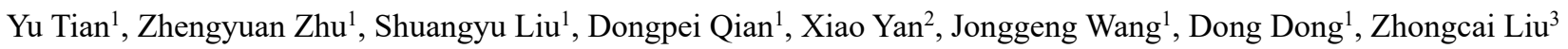 \\ ${ }^{1}$ ZheJiang Huiayun Technology Information Co., Ltd,310000,China \\ ${ }^{2}$ CUSPEA Institute of Technology, Wenzhou, Zhejiang, 325024, China. \\ ${ }^{3}$ Shanghai Makesens Energy Storage Technology Co., Ltd.,201600,China
}

\begin{abstract}
Lithium ion battery is the most widely used and reliable power source for electric vehicles. With the development of electric vehicles, the safety, energy density, life and reliability of lithium ion batteries have been continuously improved. However, in the field of vehicle power battery technology, battery monomers are combined in series and parallel to provide enough energy, but one of the major problems faced by group batteries is the consistency between battery monomers. Taking the capacity increment curve (IC curve) of lithium iron phosphate battery as the analysis tool, it is found that the characteristic peak of IC curve of different monomers in battery pack can reflect the relationship of monomer capacity. On this basis, the mathematical model is established, and the IC curve II peak characteristic point of a single cell are used as the reference to characterize the capacity of the single cell one by one. The results show that the method can be used in the normal charging process of the battery pack, and the capacity of the single cell in the battery pack can be characterized in real time during the whole life of the battery pack. It has certain research value for the ladder utilization and accurate management of battery pack.
\end{abstract}

\section{Introduction}

With the popularity of electric vehicles, power batteries have also been widely used in electric vehicle power systems, but also gradually exposed a series of problems, such as durability, reliability, safety and so on. the inconsistency between monomers after battery formation is one of the main causes of this series of problems.

lithium-ion battery capacity is one of the important performance indicators to measure battery performance. it represents the amount of electricity released by the battery under certain conditions (discharge rate, temperature, termination voltage, etc.), i.e., the capacity of the battery, usually in amperes - hours (abbreviated as $\mathrm{A} \cdot \mathrm{H}$, $1 \mathrm{~A} \cdot \mathrm{H}=3600 \mathrm{C}$ ). During the actual use of lithium ion batteries, there will be no full discharge of the battery, it is difficult to know the total capacity of the battery, thus affecting the estimation of the state (SOC) of the battery and nuclear power, and the accurate and real-time calculation of the battery capacity. It is of great significance for battery safety management.

There are many factors affecting the consistency of the battery. From the time order, the inconsistency of the single cell in the battery pack is mainly reflected in the inconsistency in the manufacturing process and the use process. Due to the immaturity of the preparation process, the state control of raw materials and the state control of production process equipment will lead to the inconsistency of single cell ${ }^{1]}$. On the other hand, the inconsistency will become more significant due to the cycle aging of the battery during use. The inconsistency of lithium battery parameters mainly refers to the inconsistency of capacity, internal resistance and open circuit voltage. Inconsistent core string and use together, there will be the following problems.

(1) capacity decreases, the core unit forms the battery pack, the capacity conforms to the "barrel principle ", the worst core capacity determines the ability of the whole battery pack $^{2}$. In order to prevent battery overcharge and over discharge, the logic of the battery management system is so set: when discharging, when the lowest monomer voltage reaches the discharge cutoff voltage, the whole battery pack stops discharging; when charging, when the highest monomer voltage touches the charge cutoff voltage, Stop charging. When charging, the small capacity of the battery must be filled first, to meet the charging deadline, the system no longer continues to charge $^{3}$. When discharging, the battery with small capacity must release all available energy first, and the system immediately stops discharging.

In this way, the small capacity of the core is always full of discharge, but the large capacity of the core has been using part of the capacity. The entire battery pack is always partially idle.

(2) life loss, similar, battery life, is determined by the core with the shortest life. Very likely, the shortest life of the core, is the small capacity of the core. Small capacity core, each time is full of release, too strong, probably the first to reach the focus of life. Has been the end of the core life, a group of welded cores, also followed by the end of 
life.

(3) internal resistance increases negative feedback, so that the high internal resistance core accelerates deterioration.

Therefore, how to diagnose the monomer inconsistency of battery pack has been an urgent problem. By analyzing the characteristic peak of capacity increment curve (IC curve) of lithium iron phosphate battery, it is found that the characteristic peak of IC curve of different monomers in battery pack can reflect the consistency between monomers ${ }^{4}$.

\section{Capacity increment curves}

Capacity increment analysis is an important method to analyze the mechanism of battery decline, and it is also an important method to study the consistency of each cell in battery pack. Its core is the battery capacity increment curve (IC curve) ${ }^{5}$. The normal charging curve of lithium iron phosphate is shown in figure 1: the horizontal axis is the charging capacity and the longitudinal axis is the battery terminal voltage. It can be seen from the diagram that the voltage rises rapidly in the early and end of the charging curve, while in the middle of the charging curve, the voltage rises slowly. In fact, most of the charging capacity is filled in the platform part ${ }^{6}$.

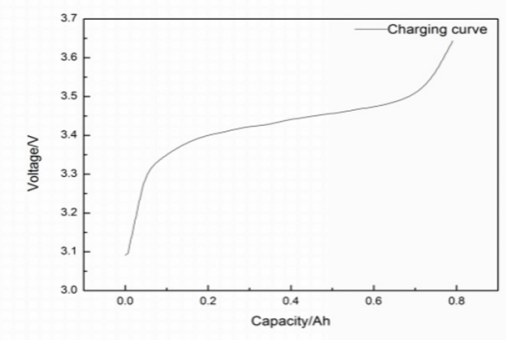

Fig1. Charging curve

The battery capacity increment curve is to process the original charging data, obtain the $\mathrm{dQ} / \mathrm{dV}$ data, and then obtain the $(\mathrm{dQ} / \mathrm{dV})-\mathrm{V}$ curve. As shown in figure 2, the advantage is that the voltage rise on the charging curve is slow, and the voltage platform region is easily observed and analyzed on the capacity increment curve ${ }^{7}$. In this paper, the IC curve of different monomer charging IC is studied, the capacity of each single cell in the series battery pack is obtained.

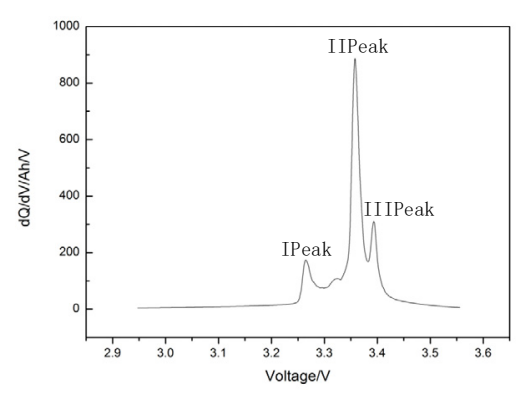

Fig2. Capacity increment curve

\section{Research on the Consistency of Li-ion Battery Packages Based on Capacity Incremental Curve}

In this paper, the voltage and current of each monomer IC curve and the eigenvalue position of the reference curve are taken as the input parameters of the algorithm model, so as to complete the real-time calculation of the capacity of all the monomers in the battery pack.

First, the charging data of the target lithium ion battery are processed, and the five-point and three-time smoothing filtering method is adopted ( 2 data before and after the smoothing filtering position are selected, a total of 5 data are selected, and the third order polynomial is used to fit the charging data of the target lithium ion battery. The capacity increment value of the position is obtained by taking out the value of the smoothing filter. The formula is

$$
\frac{\mathrm{dQ}}{\mathrm{dV}}=\frac{\Delta \mathrm{Q}_{\mathrm{k}}}{\Delta V_{\mathrm{k}}}=\frac{\mathrm{Q}_{\mathrm{k}}-\mathrm{Q}_{\mathrm{k}-1}}{\mathrm{~V}_{\mathrm{k}}-\mathrm{V}_{\mathrm{k}-1}}
$$

The characteristic peak position is the maximum position in the capacity increment curve, and the charging capacity Q1 and Q2, corresponding to the characteristic value position and the total battery capacity $\mathrm{Q}$. are recorded

The capacity coefficient between the characteristic peaks of the battery is calculated. The formula is as follows:

$$
\gamma=\frac{\mathrm{Q}_{1}-\mathrm{Q}_{2}}{\mathrm{Q}}
$$

When calculating the capacity of the $\mathrm{j}$ monomer, the charging capacity of the first eigenvalue of the IC curve is recorded $\mathrm{Q}_{1 \mathrm{j}}$, the charging capacity of the second eigenvalue of the IC curve is recorded. Thus, the total capacity corresponding to the charging curve data of all single cells is calculated. The capacity calculation formula of the $\mathrm{j}$ monomer is as follows:

$$
\mathrm{Q}=\frac{\mathrm{Q}_{1 \mathrm{j}}-\mathrm{Q}_{2 \mathrm{j}}}{\gamma}
$$

The $\mathrm{Q}_{\mathrm{j}}$ is the total capacity value corresponding to the data of the charging curve line of the $\mathrm{j}$ single cell, and the charging capacity of the $\mathrm{j}$ single cell at the first characteristic peak position. The $Q_{2 j}$ is the charging capacity of the second characteristic peak position No. $\mathrm{j}$ single cell.

Calculate the total capacity of cell pack No .1 cell:

$$
\begin{aligned}
& \text { Cap }=\frac{\Delta Q_{1}}{g}=\frac{\left|Q_{21}-Q_{11}\right|}{g} \\
& =\frac{21.56-11.576}{0.32}=31.2 \mathrm{Ah}
\end{aligned}
$$




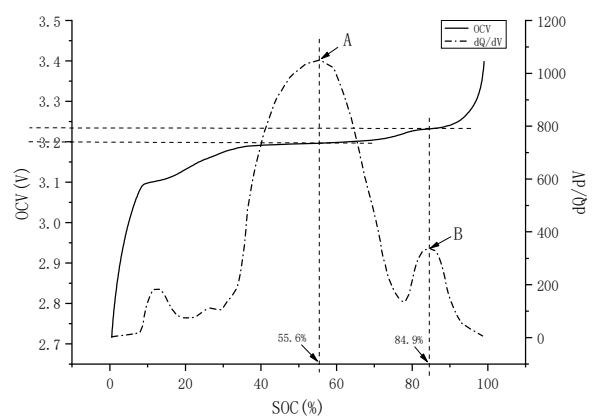

Fig3. Capacity increment curve

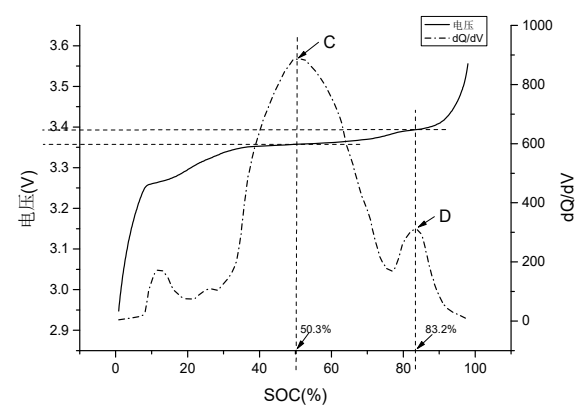

Fig4. Capacity Incremental Curve of Single Cell No .1

This paper uses the target lithium ion battery is the CATL lithium iron phosphate battery, the nominal capacity is $\mathrm{Cap}$ (initial) $=180 \mathrm{Ah}$, the battery reference curve data uses the SOC-OCV relation curve data, the data interval is 2 . The capacity value corresponding to each OCV point is calculated by formula: $\mathrm{Q}=\mathrm{SOC} * 180$.

Adopt the five-point and three-time smoothing filtering method, Five data, Using the 3rd order polynomial, Find the value after smoothing filter) find the capacity increment curve of SOC-OCV relation data, As shown in the $d Q / d V$ points in Figure 3, Figure 3 is based on SOC, And the left ordinate is OCV, battery open circuit voltage $\mathrm{dQ} / \mathrm{dV}$, is the right ordinate And the first eigenvalue position is the A point position in figure 3, Corresponding SOC is 55.6\%, Volume Q_1= Ah, 100.08 And the second eigenvalue is the $\mathrm{B}$ point in figure 3, Corresponding SOC is 84.9 per cent, Volume Q_2= $\mathrm{Ah} ; 152.82$

The relationship coefficient between the battery characteristic value and the total capacity is calculated by the following formula.

$$
\begin{aligned}
& \mathrm{g}=\frac{\Delta \mathrm{Q}}{\mathrm{Cap}_{\text {initial }}}=\frac{\left|\mathrm{Q}_{1}-\mathrm{Q}_{2}\right|}{\text { Cap }_{\text {initial }}} \\
& =\frac{|152.82-100.08|}{180}=0.293
\end{aligned}
$$

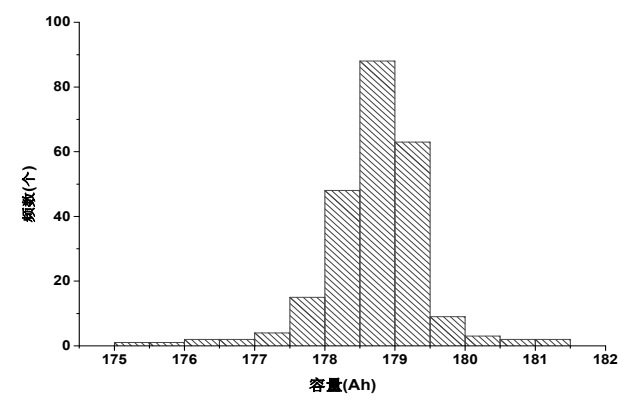

Fig5. Histogram of Single Cell Capacity

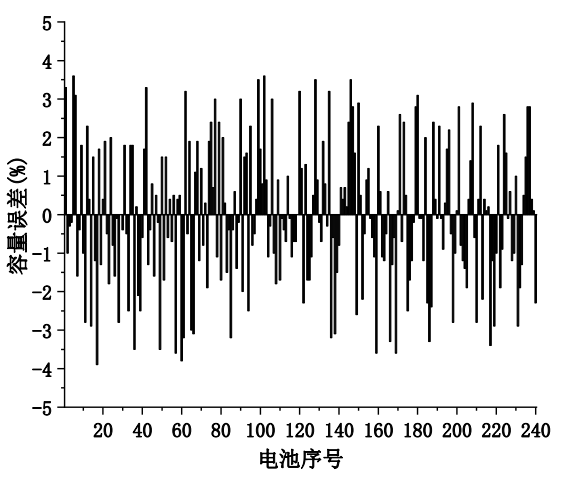

Fig6. Capacity error rate

The battery pack assembled by the target lithium ion battery is a commercial energy storage system composed of 240 single cells in series. The charging data condition of figure 3 is that the battery pack stops charging at 0.35 $\mathrm{C}, 63 \mathrm{~A}$ current to any single cell voltage of $3.6 \mathrm{~V}$, the battery pack SOC is 20 . The extraction condition of charging data is $\Delta \mathrm{V} \geq 2 \mathrm{mV}$, Extract the voltage and charge values of each cell, Using a five-point and three-time smoothing filter for the voltage and charge values of the battery, The capacity increment curve of single cell No .1 in the battery pack is shown in figure 4 . Figure 4 is based on SOC, Left ordinate is battery voltage, $\mathrm{dQ} / \mathrm{dV}$, is the right ordinate And the first eigenvalue is the $\mathrm{C}$ point in figure 4, corresponding charging capacity is Q_11=67.66 Ah. The second eigenvalue is the $\mathrm{D}$ point in figure 4 , corresponding charging capacity value is Q $21=119.2 \mathrm{Ah}$;

The same calculation method calculates the total capacity of No .2 single cell in the battery pack to the total capacity of No .240 single cell, and draws the total capacity of all single cells into histogram. Figure 5 shows the histogram of the capacity of all single cells in the battery pack.

The capacity value calculated by the invention method is compared with the real capacity value of the battery. The capacity error rate is shown in figure 6 , and the maximum capacity error is $3.86 \%$.

\section{Conclusions}

The capacity of lithium ion battery is related to the charge and discharge capacity of the battery. The capacity difference of the single cell in the series battery pack seriously reduces the performance of the battery pack. In order to better calculate the capacity of single cell in 
battery pack, this paper takes the capacity increment curve (IC curve) of lithium iron phosphate battery as the analysis tool.The capacity difference between the peak values of IC of different monomers in battery pack and the capacity value of each single cell are calculated by parameter analysis. Through experiments, the difference between the method and the actual capacity is calculated, and it is found that the maximum error between the method and the actual capacity is less than 5\%. It shows that this method can quickly calculate the capacity of single cell in battery pack, and can be applied in the normal charging process of battery pack. The capacity of single cell in battery pack can be described and analyzed in real time. It has certain research value for the echelon utilization and accurate management of battery pack.

\section{References}

1. D Qian, Z Liu, D Liu, et al. Research on consistency of Grouped lithium batteries Based on Capacity Increment Curve [C]the 2019International Conference. (2019)

2. Z.C Liu, X Yan, W Yu, et al. New Method for Determination of Health Status of Li-ion Battery [J] PST 043(001):74-76,157(2019)

3. M Xu, Z.C Liu, Yan Xiao, et al. [J]. Method for Online Detection of Internal Resistance Consistency in Capacity Increment Energy Storage Science and Technology ,008(006):1197-1203(2019).

4. W.H Lin, X Yan, Y Wang, et al. [J]. on the Characteristic Internal Resistance of Lithium Iron Phosphate Battery Power Technology 043(007):11161181,124(2019)

5. X.B Han, M.G OUYANG, L.G Lu ,et al .A comparative study of commercial lithium ion battery cycle life in electrical vehicle: Aging mechanism identification [J]. JPS, 251: 38-54 (2014)

6. M.G OUYANG, X.N FENG, X.B HAN, et al. A dynamic capacity degradation model and its applications considering varying load for a large format Li-ion battery [J]. AE,165: 48-59(2016)

7. C.R BIRKL, M.R ROBERTS, E MCTURK, et al. Degradation diagnostics for lithium ion cells $[\mathrm{J}]$. J PS,341: 373-386(2017) 